\title{
S1 Dataset
}

Each sample in the CLIPEval dataset is composed of four parts: a sentence id, a sentence extracted from the English Gigaword corpus, a polarity label and a event label. Some examples in the training set are listed in the following:

1). 1 "And it seems we can walk and run on the mountain for hours again" he added in a recent interview. NEUTRAL OUTDOOR_ACTIVITY

2). 3 "Every time I have a dispute with my wife, I shout at my sons" he told reporters in Brussels. NEGATIVE COMMUNICATION_ISSUE

3). 5 "I did it to improve my health and myself" he said. POSITIVE PERSONAL_CARE

The full dataset are available at https://alt.qcri.org/semeval2015/task9/ 